\title{
Determination of pulp necrosis based on periapical digital radiography histogram and pulp histopathology
}

\author{
Emi Khoironi*, Ria Noerianingsih Firman**, Azhari**, Fahmi Oscandar ** \\ *Department of Dental Radiology Faculty of Dentistry Universitas Hang Tuah, Indonesia \\ **Department of Dentomaxillofacial Radiology Faculty of Dentistry Universitas Padjadjaran, \\ Indonesia
}

\begin{abstract}
Introduction: Radiographic examination is needed to determine the diagnosis of pulp necrosis in addition to a clinical examination. Visual observation was limited in seeing the colour change degree and hence an effort taken by assessing the histogram value. The purpose of this study was to obtain the pulp chamber histogram pattern which reveals its grey scale value, trend, intensity average, histogram variation, and histograms maximum regional of interest (ROI) through digital periapical radiograph. Methods: This study was a descriptive study of the total of nine pulp chamber periapical radiograph data samples. The samples were divided into three groups, the $1^{\text {st }}$ group was the data taken prior to the tooth extraction, the $2^{\text {nd }}$ group was the data collected after the teeth extraction, and the $3^{\text {rd }}$ group was the data of priorly pulpless teeth. Results: There was a tendency of histogram graphic shifting to the left side, likely towards the radiolucent area on ROI of the pulp at the apical region, whilst histopathologically, a massive infiltration of a round PMN cells was found in the area. This finding supported the determination of pulp necrosis diagnose. Conclusion: The tooth with a pulp necrosis showed a tendency that led to radiolucency on periapical radiograph histogram, and histopathologic examination showed massive infiltration of a round PMN cells, thus supported the pulp necrosis diagnose.
\end{abstract}

Keywords: Pulp necrosis, periapical digital radiography, histogram graphic, histopathology

P-ISSN 1979-0201, e-ISSN 2549-6212 Available from: http://jurnal.unpad.ac.id/pjd/article/view/14300

DOI: 10.24198/pjd.vol29no3.14300

Submission: July 2017 Publishing: Nov 2017

\section{INTRODUCTION}

Dental health problems in Indonesia are still an important public health problem because the prevalence of caries and periodontal disease reaches $80 \%$ of the population. Efforts to overcome them have not given tangible results when measured with a community dental indicator. ${ }^{1}$ Caries occur due to the demineralization process occurring on the tooth surface, as well as periodontal disease occurring in periodontal membranes, bacteria entering the pulp chamber

Corresponding author: Emi Khoironi, Department of Dental Radiology Faculty of Dentistry Universitas Hang Tuah, Indonesia, Jl. Arif Rahman Hakim 150, Surabaya East Java 60111 Email:emykaha@gmail.com 
through deep caries, blood flow or through nonsterile dentistry ${ }^{2}$

The pulp is soft tissue with various cell components and extracellular matrix with the largest component being fibroblasts. The process of inflammation in the pulp tissue is allowed to cause necrosis. ${ }^{3}$ Pulp necrosis is the death of the pulp can be partial or all of the pulp involved. Clinically marked discolouration and absence of pulp response to stimulation. ${ }^{3}$

The use of $\mathrm{x}$-rays is an important part of dentistry. Radiography is often used as a reference tool to support the diagnosis of various abnormalities of teeth and jaw. In assessing the condition of the pulp, radiographic examination is needed to support clinical examination. ${ }^{4}$ The result of a radiograph produced by $\mathrm{X}$-rays penetrating an organ has a different rate, depending on anatomical density. The final feature on the film is due to the difference in X-ray absorption by body parts exposed to X-rays. This absorption depends on the volume and thickness of the tissue it passes, thus affecting the density of the image on the radiograph. Certain bone thickness will absorb more X-rays than blood or soft tissue, and they absorb more of the fat. This distinction opens the rough boundary of anatomy and pathological processes. $^{5}$

Interpretation of radiograph is the process of finding or finding all information on a radiograph by giving a black, white, and grey image. The objective is to identify the disease, to complete the information of the nature, to know the development of a disease, and to enable the diagnosis to be obtained. ${ }^{6}$ The results of the periapical radiograph examination have limitations that are only conjectural and subjective since they are two-dimensional images with three-dimensional objects. Digital radiography can produce images by numerical processing ${ }^{8}$ and quantitative analysis of the average value of greyscale pixels using digital radiographic histograms is observed by the scale of grey scale from the examination results will be obtained an objective assessment of the results of radiograph examination. The purpose of this research is to know the description of the histogram on pulp necrosis with periapical radiography and to know histopathology picture of pulp necrosis.

\section{METHODS}

The research design is descriptive research with the results obtained in the form of quantitative data. The population in this study were all patients who visited the Radiology clinic of RSGM/Dental Hospital Unpad during September-November 2013. The sample in this study was periapical radiograph taken with the parallel technique from patients with a diagnosis of pulp necrosis as seen in Figure 1.

The samples were taken based on accidental sampling method with inclusion criteria i.e. maxillary or mandibulary teeth with pulp necrosis, complete from crown to root based on clinical examination; taken by parallel technique; no other lesions on pulp chamber, such as radioopaque lesion so as not disturbing judgment; the photograph is in good quality with no error, like too dark, too bright, less contrast, less sharp, foreign body appearance or wrong position placement, stated by dentist radiology specialist in radiology. Exclusion criteria are age, gender, race, the oral condition of patient hygiene and systemic disease suffered by the patient.

The measurement scale in this study was the histogram obtained by determining the ROI of $30 \times 30$, along the pulp chamber from the apical direction. ROI obtained in one pulp chamber as much as 6 pieces, then input to the Matlab software version R2012a. The output data

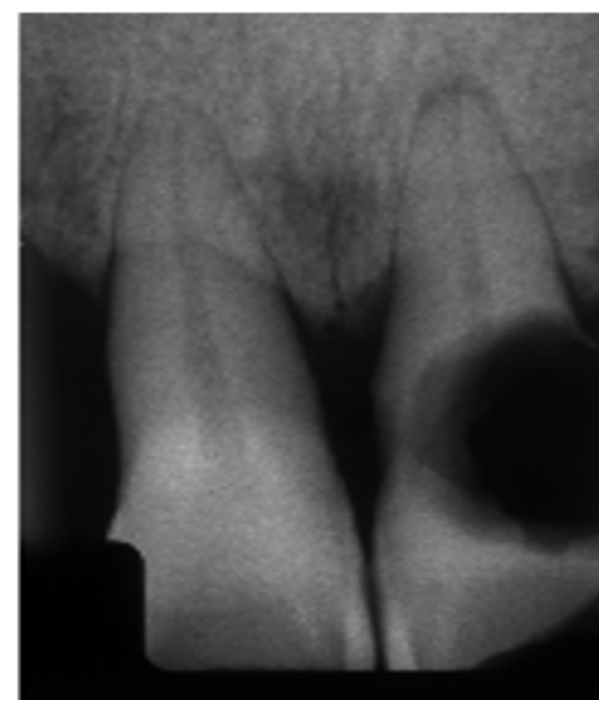

Figure 1. Diagnose of pulp necrosis in the $21^{\text {st }}$ tooth 


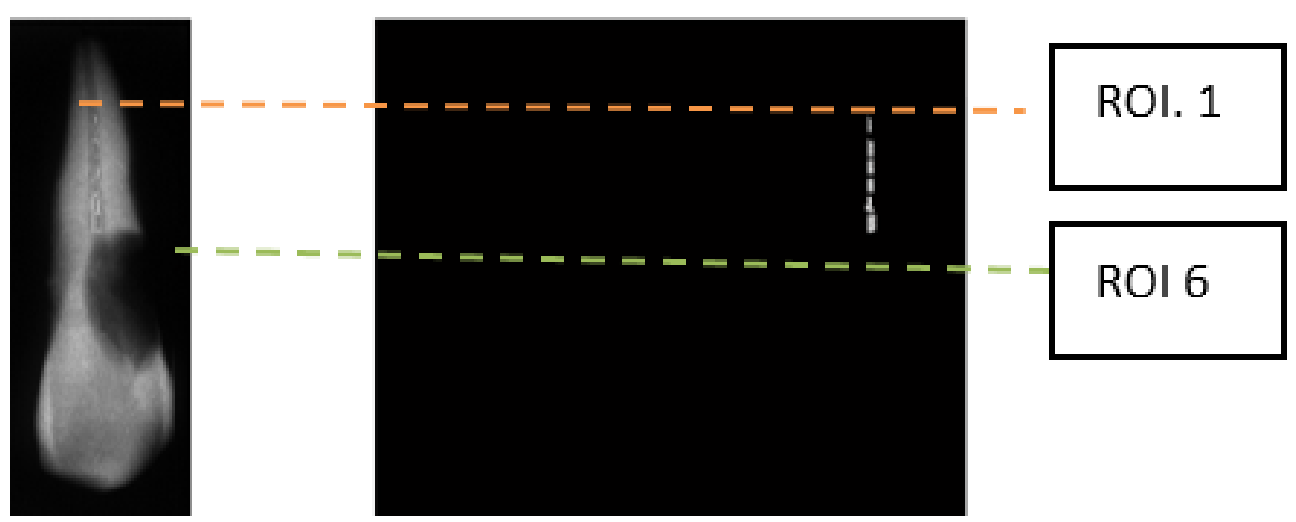

Figure 2. Cropped Image of the necrotic pulp

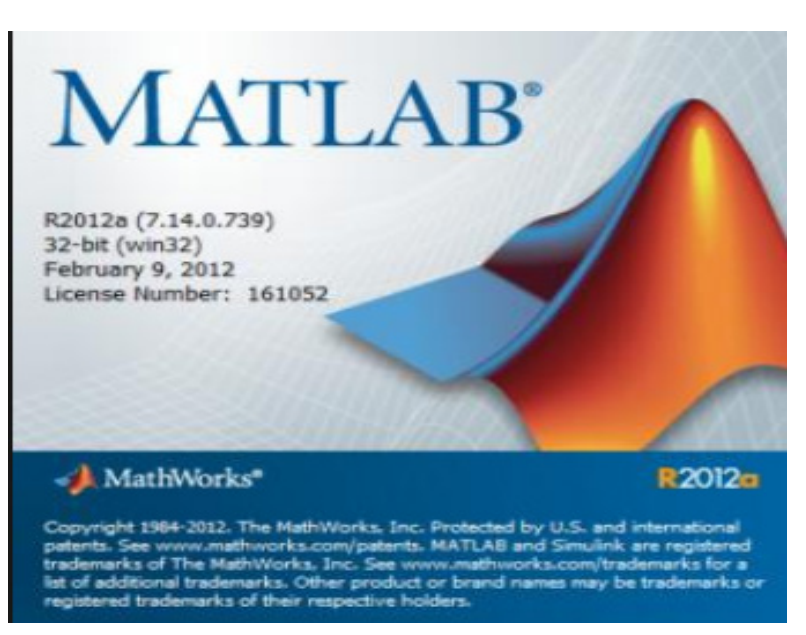

Figure 3. Matlab v.7.14.0.739

was histogram graphics, ROI intensity, variance histogram ROI, and maximum histogram ROI.

The procedure of the research through several stages, that were: Stage 1 (tooth before extraction), the teeth diagnosed with pulp necrosis were done periapical photographs with parallel technique, scanned on digital $\mathrm{x}$-ray reader $\mathrm{A} 4$ size/scanner transparent with resolution 1200 DPI (dot per inch). The radiographs result was croped with a box shape on the area to be studied as ROI. Each tooth was divided into six segments from the bottom of the pulp duct near the apical, up to the top of the pulp, to determine the difference in pulp necrosis conditions (Fig. 2). Then, Matlab Software version of R2012a was conducted, and data processing was performed (Fig. 3).

Stage 2 (tooth after being extracted with pulp): extracted maxillary anterior teeth was performed periapical radiography with parallel technique with conventional equipment, then scanned on digital X-Ray reader A4/transparent scanner with $1200 \mathrm{DPI}$ resolution, then performed the examination as in Stage 1.

Stage 3 (tooth after extraction without pulp): Extracted of maxillary anterior teeth was bur with fissure bur and removed pulp tissue then placed in glass preparation and fixation with formalin solution (formaldehyde dissolved in 1:9 aquadest). Histopathological examination was performed with eosin hematoxylin and then the preparat was photographed with microscope.

Stage 4 (tooth after extraction without pulp): maxillary anterior tooth element without pulp tissue was performed a periapical radiographic examination with parallel technique on conventional equipment, then scanned on digital $\mathrm{X}$-Ray reader A4/scanner transparent 1200 DPI resolution. Then did the examination as in stage 1.

Tools and materials used in this research was conventional $\mathrm{x}$-ray equipment, Digital X-Ray Reader A4/Transparent scanner with 1200 DPI resolution, Toshiba Portege Intel Core 13 Laptop Windows 7, and Matlab Software version of R2012a.

\section{RESULTS}

The results of histopathological examination of necrotic tooth pulp tissue from samples consist of three teeth, two cannot be checked because the sample is not representative/preparation is too small, Conducted Hematoxylin-eosin, the result is no colour difference from fat and protein composition because the sample preparation is too small. On macroscopic examination with small pulp tissue samples, brownish white. Microscopic 


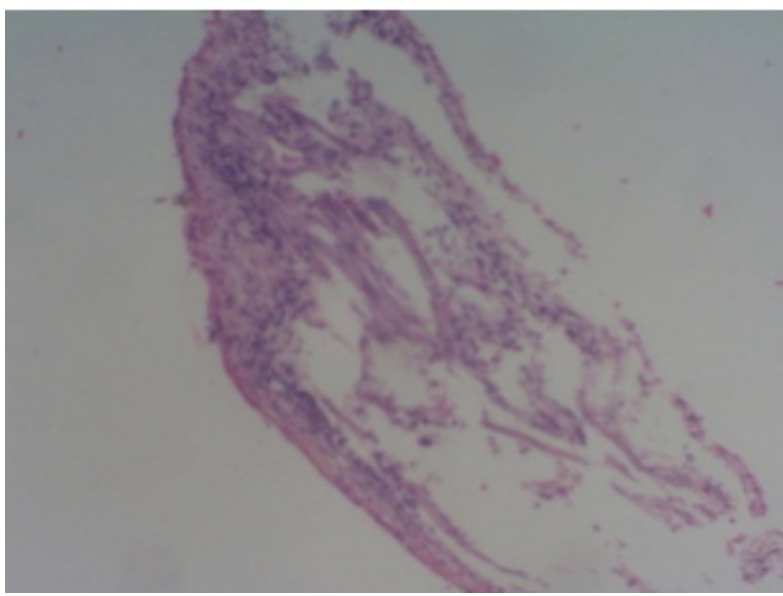

Figure 4. The histopathology of necrotic pulp of the $2^{\text {nd }}$ tooth
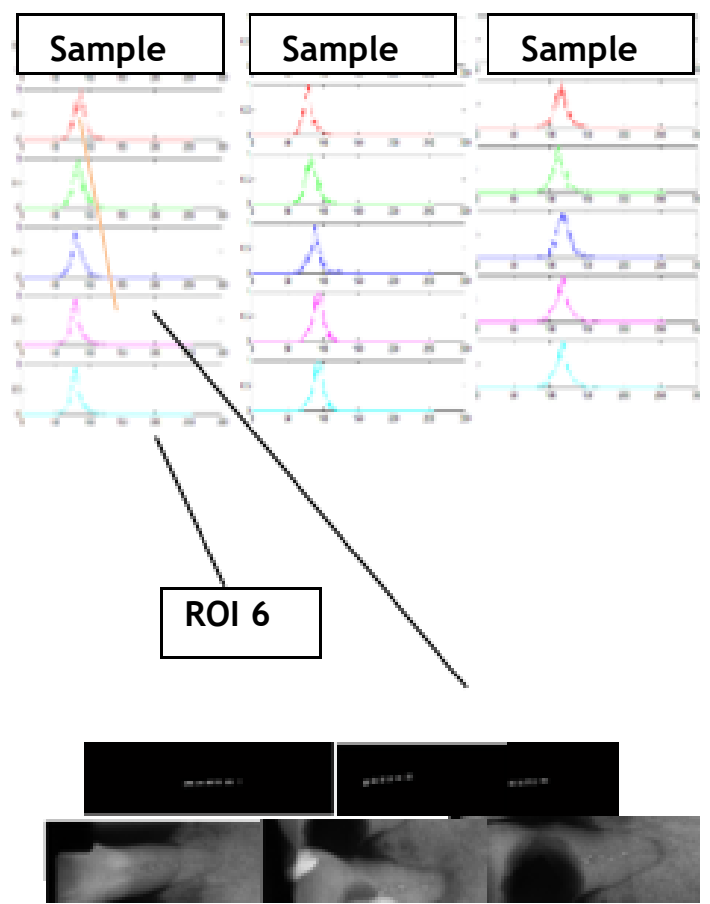

ROI 1

Figure 5. Tooth histogram prior to extraction examination there is massive infiltration of PMN round cell.

The histogram pattern, the maximum value of the ROI histogram of samples 1, 2, and 3 on the tooth before it was pulled, shows the cervical pattern, the maximum peak of the histogram getting to the left of l, which means it is closer to the lowest value of the radiolucent.

The histogram pattern of the maximum value of the histogram of samples 1, 2 and 3 on the tooth after being pulp removed, the pulp chamber shows an increasingly cervical histogram pattern, the maximum peak of the histogram getting to the left of each sample, which means closer to the lowest value of the radiolucent.

The average intensity pattern of each ROI In the tooth before it is pulled, the more coronal radiolucent seen in table 1 (from point 0.45 to point 0.3 ), after revoked the pattern looks down then on the third ROI up to the 5th ROI, from radiointermediate in the first ROI position (apical) to radiolucency (approached coronal) After unplugged without a pulp seen pattern from apical ROI to coronal down means from radio-intermediate to more radiolucent (from point 0.6 to point 0.4 ).

The variance histogram of the sample ROI of 1 tooth before it was removed ranged from 0.2335 to 0.2272 , the tooth after being removed with pulps ranged from 0.2724 to 0.2136 and the tooth after being removed without pulp between 0.2479 to 0.1723 . Obtained histogram variance data is not much varied (If the width of the histogram variance the more variants, the more unpolluted the pulp content.

Maximum histogram of dental ROI before revoked on the first $\mathrm{ROI} 0.3804$ graph decreased until the 5 th ROI 0.3098 , visible pattern of numbers

Table 1. The average of intensity, histogram variance, and maximum histogram prior to the tooth extraction

\begin{tabular}{llcccccc}
\hline & & ROI 1 & ROI 2 & ROI 3 & ROI 4 & ROI 5 & ROI 6 \\
\hline Intensity average & Sample 1 & 0.4249 & 0.3875 & 0.3789 & 0.3719 & 0.3642 & 0.3760 \\
& Sample 2 & 0.3942 & 0.3601 & 0.3759 & 0.3922 & 0.4069 & 0.3991 \\
& Sample 3 & 0.4740 & 0.4820 & 0.4715 & 0.4949 & 0.4886 & 0.4605 \\
Histogram variance & Sample 1 & 0.2335 & 0.2183 & 0.2339 & 0.2060 & 0.2095 & 0.2272 \\
& Sample 2 & 0.2120 & 0.2166 & 0.2413 & 0.1969 & 0.2393 & 0.1818 \\
& Sample 3 & 0.2291 & 0.2117 & 0.2111 & 0.2487 & 0.2182 & 0.2235 \\
& Sample 1 & 0.3804 & 0.3490 & 0.3333 & 0.3098 & 0.3098 & 0.3451 \\
& Sample 2 & 0.3647 & 0.3176 & 0.3255 & 0.3490 & 0.3725 & 0.3490 \\
\hline & Sample 3 & 0.4392 & 0.4510 & 0.4392 & 0.4549 & 0.4588 & 0.4392 \\
\hline
\end{tabular}


decreased, meaning the first ROI of the apical part to the coronal of the pulp getting radiolucent. teeth after being pulled out with pulp from 0.2724 down until the fourth ROI of 0.1931 , the fifth ROI pattern rises then the sixth ROI down 0.2136 and the tooth after unplugged without pulp between 0.2479 to 0.1723 . pulp obtained ranged from 0.0274 to 0.2136 . histogram variance data does not vary much.

\section{DISCUSSION}

Pulp inflammation causes the tissue to undergo central disintegration thus forming a liquefaction necrosis region due to a lack of collateral circulation and a rigid dentin wall. This condition interferes with inflammatory drainage and increased tissue pressure increases, resulting in uncontrolled destruction until all pulp tissues are necrotic.

The rate of progression of liquefaction necrosis varies. Speed can be related to network capability. The necrotizing region contains irritants from tissue and microbial destruction, both anaerobic and aerobic.

Results of histopathological examination of pulp tissue from three tooth samples, two cannot be examined because the sample is too small, marked with no visible colour difference of fat and protein structure when given eosin hematoxylin staining. characterized by no visible colour difference from fat and protein structures when stained with hematoxylin-eosin.

Carnerio et al. ${ }^{9}$ in his study said the average grey scale can be used to look at caries remineralization, a smaller value for demineralization. This study mention that the minimum histogram value lies on the grey scale 50 and the highest histogram at number $200 .{ }^{9}$ Markvart et al. ${ }^{10}$ in his study stated that the accumulated increase in mineral deposits occurred in teeth with smaller pulp cavities compared with larger pulp cavities in addition to composition factors of pulps with different densities. As well as a barrier factor from absorption of $\mathrm{x}$-rays in the oral cavity than outside the oral cavity. ${ }^{10}$

The average of tooth pulp intensity before it is pulled increasingly to the apical closer to zero. Grossman said that the end result of pulp decomposition is hydrogen sulphide, ammonia, fatty substance, ptomaine, water and carbon dioxide and further results are indol, skatol, putrescine, and cadaverin. ${ }^{3}$ Yoon et al. ${ }^{12}$ stated that dry pulp tissue consisted of $5.7 \%$ calcium, $3.5 \%$ phosphorus, and $11.7 \%$ are nitrogen, increasingly to the more radiolucent apical, where in the coronal portion contained more air so that the density was lower than the apical region containing the end result of pulp decomposition. ${ }^{12}$

The average intensity of each ROI on a single tooth sample before being unplugged, unplugged with pulp and unplugged without pulp, shows an average pattern of intensity patterns increasingly coronal to radiolucent. This means the demineralisation process runs from coronal to apical or the process of necrosis goes from coronal to apical. The average ROI intensity in all tooth samples before removal compared to the teeth that have been removed and the teeth removed and pulp is removed on each ROI as measured from the apical part of the pulp approaching to the coronal down, meaning the coronal density further decreases so that more radiolucent, pulp density in the region approximates the maximum apical so that more radiopaque in this study is shown by the average number of ROI intensity on the apical pulps higher approximation value.

The variance of the histogram of ROI in one tooth sample was compared before it was revoked after it was removed with pulp and tooth after unplugged without pulp, the data of histogram variance did not vary much. Similarly, the comparison of the three samples, namely the teeth have not been revoked, pulled out with pulp and unplugged without pulp not too varied. The maximum ROI histogram is the highest value in the one ROI that most often appears or how many of the most frequent intensities appear, from the histogram graph, the higher the maximum value of the histogram of the high value. These varying values indicate the difference in the rate of the outcome of the pulp necrosis condition occurring on the pulp. This is related to the pathophysiological process of pulp necrosis.

Maximum comparison of the histogram of ROI on one tooth sample before revoked at first ROI $((0.3804)$ shows highest peak pattern of histogram chart decreased until ROI to five $(0.3098)$, meaning the first ROI of the apical part towards coronal of pulp increasingly to left that is more radiolucent. Teeth after being pulled out with pulp (0.2724) 
down to the fourth ROI (0.1931), meaning the first $\mathrm{ROI}$ of the apical part to the coronal of the pulp (ROI four) the radiolucent, meaning the density progressively decreases to the coronal gear after being unplugged without pulp from the first ROI $(0.6118)$ decreases until the ROI of six $(0.3294)$ means the top of the graph (maximum value) of the histogram gascic the more the coronal to the radiolucent Graph The maximum histogram of ROI of all tooth samples prior to repeal, after being pulverized and after unplug pulse measured from first ROI until ROI six shows the pattern approaching zero means the density is decreasing in the coronal section the comparison of all samples before removal, after unplug and after pulling, the pattern shows more trend to the right ie more radiopak on the teeth after unplug without pulp, this is due to formalin immersion or the presence of formalin fluid on the tooth after it is unplugged without pulp formalin fluid). Water has a larger density of 1.0 compared to the air 0.000129 , which means here that water or liquids have more density or mass than gas or air. In accordance with the nature of absorption, the lower the atomic number of an object the greater the penetration of the X-ray and the greater the atomic number, the greater its absorption. ${ }^{12,13}$ So that radiographs result more radiopaque on the teeth after soaking formalin.

Differences in these variations of value are associated with an X-ray interaction when it comes to a material, in which case the material used is a pulp with a diagnosis of pulp necrosis. Each atom number ( $Z$ ) affects the thickness of the resulting image, the greater the atomic number, the number of electrons attached to each electron shell will be greater. This greatly affects the radiodensity and contrast of the resulting image. The lower the atomic number of an object or matter, the greater the penetration of the X-ray, the greater the atomic number, the greater its absorption. ${ }^{12,13}$

The pulp decomposition of each compound has different atomic number. Consecutively for the number of hydrogen atoms, carbon, nitrogen, and oxygen are 1, 6, 7, and 8 . The atomic number (commonly denoted by $Z$ ) is the number of protons present in the atomic nucleus. The number of protons is equal to the number of electrons in the compound. The atomic number and the number of electrons in the compounds are related to the interaction of light on the object, in this study is a pulp that has been necrosis with different decomposition results.

The density is affected by the material, the physical characteristics of the contrast material from the water of the effective atomic number (z) 7.42 , density $1.0 \mathrm{gr} / \mathrm{cm}^{3}$; fat atomic number effective (z) 5.92, density 0.91; air of effective atomic number (z) 7.64, density 0.00129 ; calcium effective atomic number (z) 20.0, density 1.55; effective atomic number $(z) 7.46$, density 1.0 $\mathrm{gr} / \mathrm{cm}^{3}$; barium effective atomic number $(\mathrm{z})$ 56.0 , density $3.5 \mathrm{gr} / \mathrm{cm}^{3}$; iodine effective atomic number (z) 56.0 , density $3.5 \mathrm{gr} / \mathrm{cm}^{3}$. According to the nature of the absorbs of $\mathrm{x}$-rays when the mass of the material is greater, the absorption power of the $x$-rays will also be greater, ${ }^{14}$ so that grayscale pulp necrosis is more radiolucent.

\section{CONCLUSION}

The tooth with a pulp necrosis showed a tendency that led to radiolucency on periapical radiograph histogram, and histopathologic examination showed massive infiltration of a round PMN cells, thus supported the pulp necrosis diagnose.

\section{REFERENCES}

1. National Institute of Health Research and Development (NIHRD). Indonesia basic health research (RISKESDAS) 2007-2008. Jakarta: Ministry of Health Republic of Indonesia; 2008.

2. Rajendran R. Shafer's texbook of oral pathology. $6^{\text {th }}$ ed. New Delhi: Reed Elsevier India Pvt. Ltd.; 2009. p. 682-3.

3. Grossman LI, Seymour O, Del Rio CE. Endodontic practice. $11^{\text {th }}$ ed. Philadelphia: Lea \& Febiger; 1988. p. 40-83.

4. Frommer $\mathrm{HH}$, Stabulas-Savage JJ. Radiology for the dental professional. $9^{\text {th }}$ ed. St. Louis: Mosby-Elsevier; 2011.

5. Ingle JI, Simon JHS, Walton RE, Pashley DH, Bakland LK, Heithersay GS, et al. Pulpal pathology: its etiology and prevention. In: Ingle JI, Bakland LK. Endodontics. $4^{\text {th }}$ ed. Hamilton: BC Decker Inc.; 2002. p. 95-174.

6. Novelline RA. Squire's fundamentals of radiology. $6^{\text {th }}$ ed. Cambridge: President \& Fellows of Harvard College; 2004. 
7. Attwood D, Sakdinawat A. Soft X-rays and extreme ultraviolet radiation: Principles and application. $2^{\text {nd }}$ ed. Cambridge: Cambridge University Press; 2016. p. 2.

8. Nelson SJ. Wheeler's dental anatomy, physiology, and occlusion. $9^{\text {th }}$ ed. St. Louis: Saunders-Elsevier; 2010. p. 209, 223.

9. Carneiro LS, Nunes CA, Silva MA, Leles CR, Mendonca EF. In vivo study of pixel grey measurement in digital subtraction radiography for monitoring caries remineralization. Dentomaxillofac Radiol Feb 2009;38(2):73-8. DOI: $10.1259 / \mathrm{dmfr} / 15857365$.

10. Markvart M, Bjørndal L, Darvann TA, Larsen P, Dalstra M, Kreiborg S. Threedimensional analysis of the pulp cavity on surface models of molar teeth, using x-ray micro-computed tomography. Acta Odontol Scand Mar 2012;70(2):133-9. DOI: 10.3109/00016357.2011.600707.

11. Yoon SH, Brudevold F, Smith FA, Gardner DE. Flouride, calcium, phosphate, ash, and water content of human dental pulps. J Dent Res Jul 1965;44:696-700. DOI: $10.1177 / 00220345650440041601$.

12. Whaites E. Essentials of dental radiography and radiology. $4^{\text {th }}$ ed. London: Churchill Livingstone; 2007.

13. White SC, Oral radiology: principles and interpretation. $7^{\text {th }}$ ed. St. Louis: MosbyElsevier; 2014.

14. Sprawls P Jr. The physical principles of medical imaging. $2^{\text {nd }}$ ed. Madison: Sprawls Educational Foundation; 1995. 